\title{
CORRELATED SIMULTANEOUS PHASON JUMPS IN AN ICOSAHEDRAL Al-Mn-Pd QUASICRYSTAL
}

\author{
G. Coddens $^{1}$, S. Lyonnard ${ }^{1}$, B. Hennion ${ }^{1}$ and Y. Calvayrac ${ }^{2}$
}

\author{
Ph.D. thesis of S. Lyonnard \\ ${ }^{1}$ Laboratoire Léon Brillouin (CEA-CNRS) \\ ${ }^{2}$ C.E.C.M./C.N.R.S., 15, rue G. Urbain, F-94407 Vitry Cedex, France
}

The relationships between quasicrystals and other incommensurately modulated crystals (IC) have many interesting theoretical aspects. An obvious link is that both can be derived from a common description based on an embedding in a higher-dimensional superspace that contains a periodic lattice of so-called atomic surfaces. The superspace cut-and-projection algorithm permits a group-theoretical classification of all possible structures. Hyperspace crystallography leads also to the possibility of an attractive analogy within the realm of lattice dynamics based on the introduction of the notion of " phasons " in addition to the usual phonons. Here we stumble onto a first real difficulty. Once we go beyond one-dimensional structures, there are very important differences in the topology of the atomic surfaces between QC and IC. This has been pointed out by many authors, and turns the subject into a really subtle issue. Generally spoken, the atomic surfaces in QC are not continuous ${ }^{[1]}$.

Based on these studies it was anticipated that "phasons" in QC would not be collective propagating modes as in IC but rather atomic jumps ${ }^{[2]}$, that can be visualized by configuration flips within Penrose-like tiling models. Ensuing experimental studies confirmed this picture. In our current understanding an atomic jump is a stochastic single-particle process. The phonon heat bath produces a fluctuating environment that from time to time will open a low-energy gateway that is prosperous for a jump. Starting from this conceptual image that thrives on disorder, it is hard to imagine an orderly concerted choreography of simultaneous jumps of two or more atoms. This only stresses the fact that, although they are both materialized by a sliding of the cut in superspace, phasons in QC and IC should correspond to very different, antipodal types of dynamics. Nevertheless, this poses a number of small problems in QC. First of all, the (anomalous) temperature dependence of the (quasielastic) neutron-scattering signal that reveals the existence of the hopping does not tally with the description we gave above of the jump process in terms of a phonon bath ${ }^{[3]}$. Secondly, tile flips in real, i.e. not mono-atomic structural models entail in general several simultaneous atomic jumps. It was therefore inferred that the elementary phason building brick would rather be the atomic jump than the tile flip. In triple-axis neutron-scattering experiments on a large single-grain sample of the icosahedral phase Al-Mn-Pd, we came across some evidence that seems to challenge this common-sense based paradigm. In fact, we found a $\mathbf{Q}$-dependence of the quasielastic signal that we are only able to explain by assuming that two (or more) atoms jump simultaneously keeping their separation vector fixed.

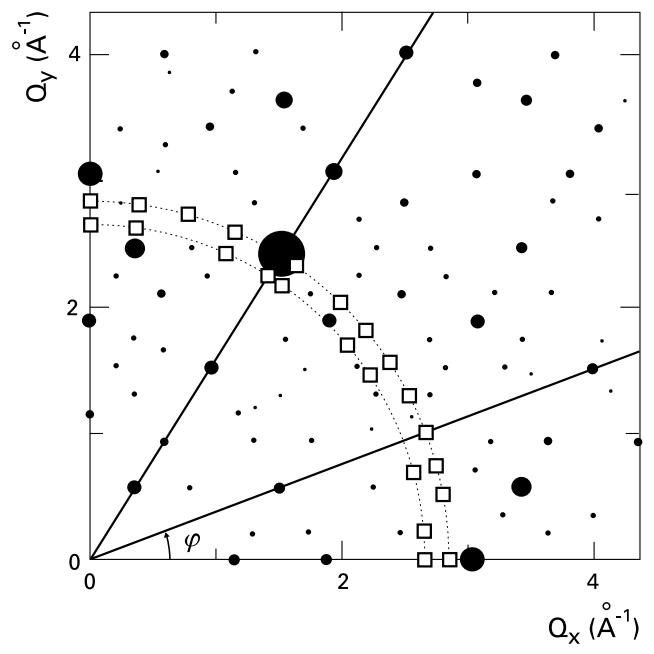

Figure 1. Binary scattering plane. The sizes of the full circles represent the intensity of the Bragg peaks. The locations of the various constant- $Q$ scans have been drawn: they were all made in the $k_{f}=1.64 \AA^{-1}$ configuration. The angle $\varphi$ is defined with respect to $Q_{x}$

These experiments were performed with the coldneutron double-monochromator triple-axis spectrometer $4 \mathrm{~F} 2$ of the LLB in the fixed $\mathrm{k}_{\mathrm{f}}=1.64 \AA^{-1}$ and $\mathrm{k}_{\mathrm{f}}=$ $1.96 \AA^{-1}$ configurations. The loci of some of the constant- $Q$ energy scans in the binary scattering plane of the QC are indicated in Fig. 1, which also shows the intensities of the most prominent Bragg peaks. The choice of the binary plane allows to explore all types of symmetry axes (2-, 3-, and 5-fold) of the QC in one set-up. The $3 \mathrm{~cm}^{3}$-sized single-grain sample has been grown by the Czochralsky method. 
A typical data set is featured in Fig. 2 together with a fit based on a Lorentzian quasielastic signal convoluted with the Gaussian resolution function. Also included in this fit are the elastic peak and a linear incoherent phonon background. The Q-dependence of the quasielastic intensity for $Q=2.85 \AA^{-1}$ is displayed in Fig. 3. It is strongly anisotropic. In a simple singleparticle model in the white-noise approximation for atomic jumps between two sites separated by jump vectors $\mathbf{d}_{\mathrm{j}}$ along a $\mathrm{m}$-fold axis of the $\mathrm{QC}$, the quasielastic intensity should follow an (incoherent) structure factor:

$$
S_{q . e l}(\mathbf{Q})=\sum_{j=1}^{30 / m} \frac{1}{2}\left[1-\cos \left(\mathbf{Q . d}_{j}\right)\right]
$$

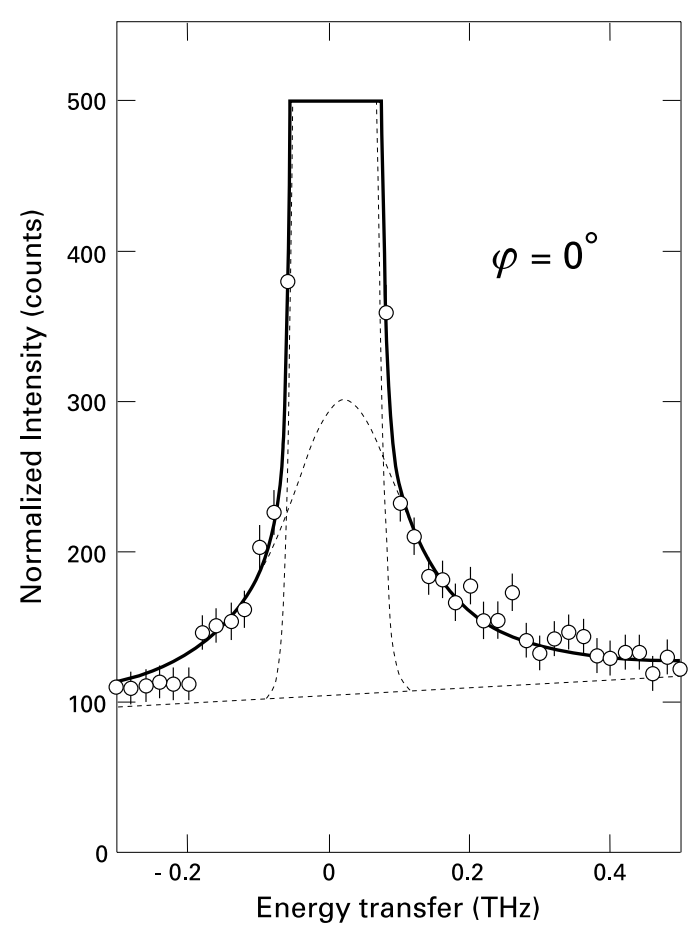

Figure 2. Typical constant- $Q$ scan with fit. The data shown here correspond to $|Q|=2.85 \AA^{-1}$ and $\varphi=0^{\circ}$.

The sum allows for the fact that for jumps along $\mathrm{m}$-fold directions there are $30 / \mathrm{m}$ symmetry-related jump vectors. If the direction of the jump is not along a symmetry axis, then the sums will have to extend over the whole icosahedral group. (This corresponds to the case $\mathrm{m}=1$ ). The quasielastic and elastic intensities obey a sum rule such that the incoherent elastic structure factor is obtained from Eq.(1) by changing the sign in front of the cosine term:

$$
S_{e l}(\mathbf{Q})=\sum_{j=1}^{30 / m} \frac{1}{2}\left[1+\cos \left(\mathbf{Q . d}_{j}\right)\right]
$$
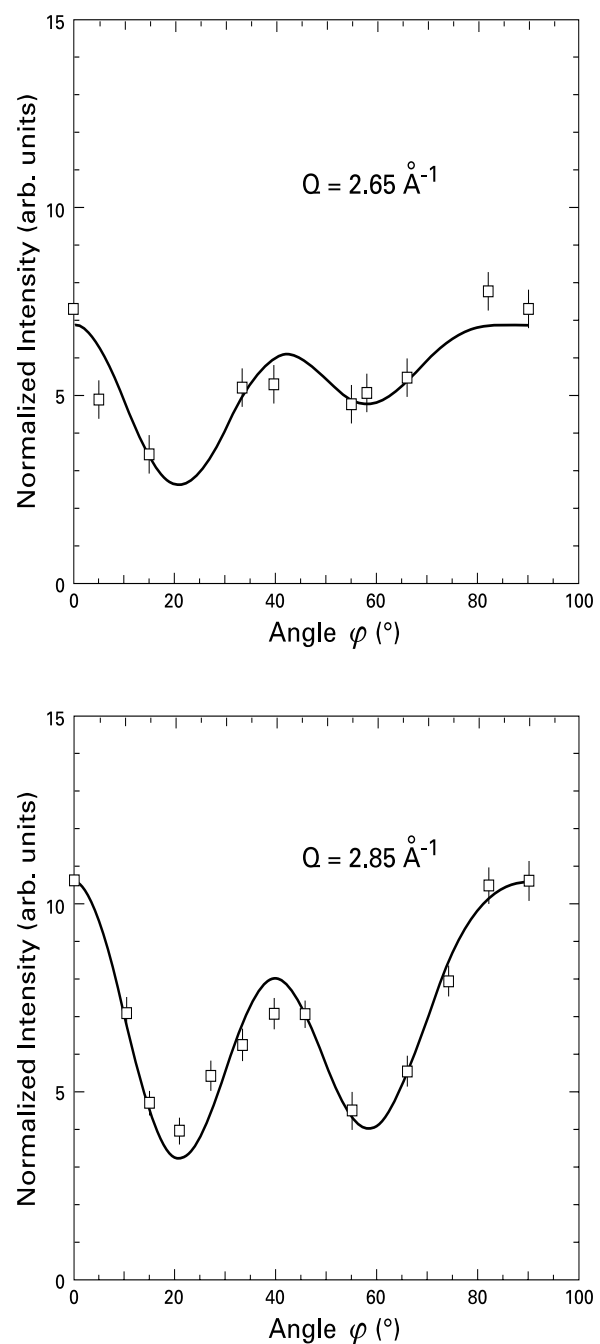

Figure 3. Quasielastic intensities for $|Q|=2.65 \AA^{-1}$ and $|Q|$ $=2.85 \AA^{-1}$. A fit with model function (2) is also shown. (Data collected with a second sample, both in $k_{f}=1.64 \AA^{-1}$ and $k_{f}=1.97 \AA^{-1}$ set-ups, bear out the reproducibility of these results).

The quasielastic structure factors embodied by equation (1) all exhibit an isotropic first local maximum in $Q$, as can be appreciated from Fig. 4 for two-fold jumps. This remains true if the direction of the jump is not a symmetry axis. This is quite at variance with the experimentally observed data which do not display such a spherical shell of maximum intensity and on the contrary exhibit their strongest anisotropy at low $Q$. We should point out that none of the three models $(m=2 ; \quad m=3 ; \quad m=5)$ expressed by equation (1) can reproduce the peak/valley-ratios that occur in Figure 3. This means that one needs another type of model. By some serendipity we found out that the data shown in Fig. 3 are perfectly described by equation (2) for $m=3$ and $\mathrm{d}=3.85 \AA$. Among the (idle) lines of thought we followed in our attempts to come to grasps with this alienating finding, we can mention models as proposed 
by Katz and Gratias ${ }^{[4]}$ and down-sized versions of Elser's escapement model ${ }^{[4]}$. The failure to represent our data by such models helped us to realize where the minus signs in front of the cosines in equation (1) come from. Each time an atom jumps from A to an empty site $\mathrm{B}$, the neutron-scattering contrast between $\mathrm{A}$ and $\mathrm{B}$ is inverted, which amounts to a $\pi$-flip of its phase. Therefore, the only way to obtain a plus sign is by designing a model that preserves the contrast despite the occurrence of the jump. This can only be achieved by admitting that two atoms in $\mathrm{A}$ and $\mathrm{B}$ are jumping simultaneously to $A^{\prime}$ ' and $B^{\prime}$ 'respectively, whereby the vectors $\mathrm{AB}$ and $\mathrm{A}^{\prime} \mathrm{B}^{\prime}$ are equipollent. This leads to expressions of the type

$$
\mathrm{S}_{\mathrm{q}, \mathrm{el}}(\mathbf{Q})=\sum_{\mathrm{j}=1}^{30 / \mathrm{m}} \frac{1}{\mathrm{~m}}\left[1-\cos \left(\mathbf{Q . d} \mathbf{d}_{\mathrm{j}}\right)\right]\left[1+\cos \left(\mathbf{Q} . \mathbf{s}_{\mathrm{j}}\right)\right] \text {, where }
$$

the correlation vector $\mathbf{s}_{\mathrm{j}}$ is analogous to the separation vector $A B$. The $\left[1-\cos \left(\mathbf{Q} . \mathbf{d}_{j}\right)\right]$ parts are responsible for the first radial maximum, while $\left[1-\cos \left(\mathbf{Q} . \mathbf{s}_{\mathbf{j}}\right)\right]$ terms entail a modulation of this local maximum that otherwise would have been an isotropic spherical shell. In our data we thus have $\mathrm{s}_{\mathrm{j}}=3.85 \AA$, while $\mathrm{d}_{\mathrm{j}}$ cannot be determined. At once, this means that phasons in QC could nevertheless share some collective character with propagating phason modes that are typical of IC. We must admit that such collective motion clashes with our Weltanschauung, as we were strong believers of the heat-bath-driven scenario outlined above. We must signal that the description of the data by Equation (2) breaks down at higher $Q$-values, where we are unable to explain the observed intensities. At 2.65 and at $2.85 \AA^{-1}$ we are so lucky to find ourselves in the small- $Q$ limit, where the largest characteristic distance within the dynamics can be discerned in an isolated fashion, free from the obfuscating presence of signals corresponding to shorter length scales. We think that this novel result constitutes an important step towards the elucidation of the physical nature of phasons in QC.

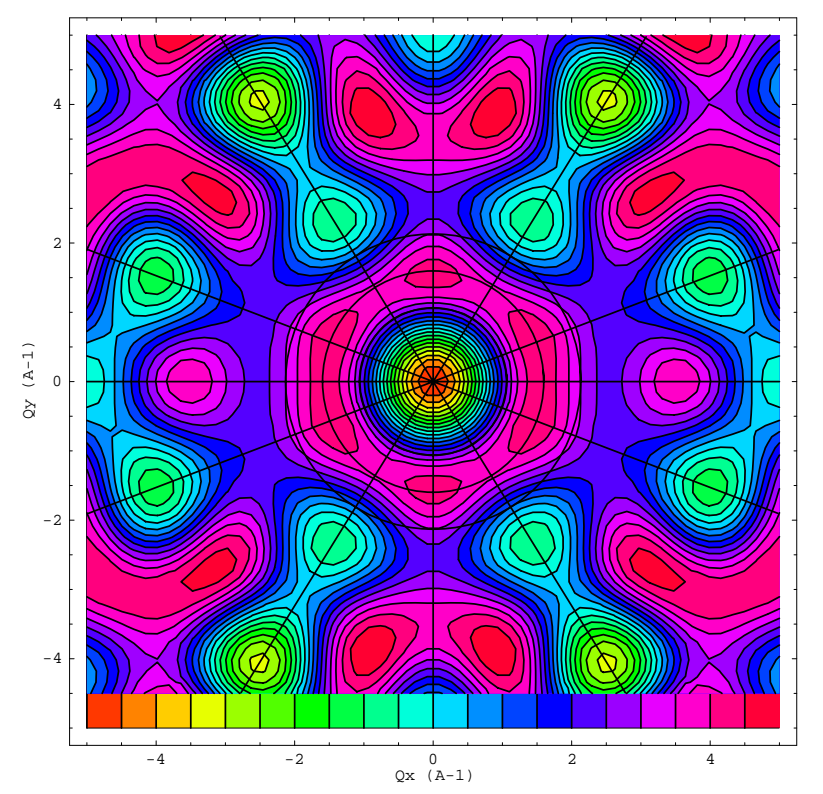

Figure 4. Contour plots for the quasielastic intensity, calculated in a model with two-fold jumps according to Equation (1), $m=2$. Note the almost isotropic first maximum of the intensity around $Q=1.5 \AA^{-1}$. The color code is shown at the bottom of the Figure, going from left to right, e.g. the first color on the left codes numerical values between 0 and 1.

\section{References}

[1] L. S. Levitov, Quasicrystals, The State of the Art, ed. D. P. DiVincenzo and P. J. Steinhardt, Directions in Condensed Matter Physics, Vol. 11, (World Scientific, Singapore, 1991) p. 239.

[2] G. Coddens, S. Lyonnard, and Y. Calvayrac, Phys. Rev. Lett. 78, 4209 (1997).

[3] A. Katz and D. Gratias, J. Non-Cryst. Solids 153 \& 154, 187 (1993)

[4] V. Elser, Phil. Mag. A 73, 641 (1996) 
